\title{
Anesthesia in patients with neuromuscular disorders
}

\section{Anestesia em Doenças Neuromusculares}

\section{Vincenzo Tegazzin}

Head of MH Laboratory, Department of Anesthesia and ICU, S.Antonio University-Hospital, Padua, Italy.

The scientific progress in molecular medicine, genetic and physiopathology of skeletal muscle junction make over the knowledge on the cause of muscle disorders (NMD) and on molecular action of anaesthetic drugs.

To obtain general anaesthesia (narcosis, muscle relaxation, analgesia) more drugs are required, which act, trough specific receptors, on the brain and on the neuromuscular junction. Therefore, the knowledge of the patho-physiology of the muscle plays an important rule in the anaesthesiological practice.

However, the complications of anesthesia in patients affected with muscle diseases could be divided in two groups: those derived from triggering agents and attributed to depolarizing muscle relaxants and volatile anesthetics (type I complications-rhabdomyolisis, hyperpotassiemia, cardiac arrest and malignant hyperthermia) and those derived from the well known general side effects of the anesthetics, namely respiratory and cardiac depression (type II complications) ${ }^{1}$.

Therefore, the aim of anaesthesia is to be "safe anaesthesia-avoiding trigger agents-" and this is an appropriate condition particularly for the patients with NMD that can suffer from cardiomyopathy, restrictive lung disease, altered metabolism, skeletal dismorphism with difficulty in airway management, altered pharmacodynamics and pharmacokinetic effects of the anaesthetic drugs.

From the literature, in the anesthetized patients with NMD the multimodal approach seems to be the more satisfactory both in adult and in the children ${ }^{1}$. Another condition that must be controlled particularly during general anesthesia is the titration of anesthetic agents, taking in mind that more than 250 drugs have an effect on neuromuscular transmission and hence interfere pharmacodynamically with muscle relaxant ${ }^{2}$.

For this reason, an accurate monitoring of $\mathrm{PA}, \mathrm{ECG}, \mathrm{ETCO} 2, \mathrm{SaO} 2$, and temperature, and the availability of a trolley with specific drugs and equipment for airway management and of an experienced anesthetist is needed. 
Another important issue concerns patients that are "suspected myopathic or malignant hyperthermic" so that, if not in emergency situations, a good examination before anesthesia should include:

1) deep anamnesis,

2) well documented reports, serum enzymes tests, specialists consulting (neurology, cardiology, respiratory),

In case of emergency situation, a general anesthesia without depolarizing muscle relaxant and halogenated is recommended, followed by proper monitoring.

\section{References}

1. Melloni C. Effects of intravenous anaesthetics on patients with muscle disease Proceedings of "Neuromuscular Diseases and Anaesthesia meeting", 2004, (in press).

2. Argov Z, Mastaglia FL. Disorders of neuromuscular transmission caused by drugs. N Engl J Med 1979; 301:409-413. 